\title{
Suplementación de selenio sobre el rendimiento reproductivo, productivo, concentración tisular y actividad de glutatión peroxidasa en cerdas y lechones: un metaanálisis
}

\author{
Selenium supplementation on reproductive and productive performance, \\ tissue concentration and glutathione peroxidase activity in sows \\ and piglets: a meta-analysis
}

\author{
Jimmy Quisirumbay-Gaibor ${ }^{1,2,3}$, Carlos Vílchez Perales ${ }^{1}$
}

\section{Resumen}

Se evaluó el efecto de la suplementación de selenio sobre el rendimiento reproductivo y productivo, concentración tisular y actividad de GSH-Px en cerdas y su progenie mediante un estudio de metaanálisis. Se utilizaron 12 artículos que incluyeron 4698 animales. Bajo el modelo de efectos aleatorios se determinó tamaño de efecto, heterogeneidad, meta-regresiones y sesgo de publicación. Las variables reproductivas no se vieron afectadas por la fuente de selenio. El peso del lechón al nacimiento fue superior ( $\mathrm{p}=0.031$ ) en cerdas que recibieron selenio inorgánico (SeI) $1.47 \mathrm{vs} 1.46 \mathrm{~kg}$ de la fuente orgánica $(\mathrm{SeO})$. El peso del lechón al destete fue $135 \mathrm{~g}$ superior $(\mathrm{p}=0.105)$ en las cerdas del grupo $\mathrm{SeO}$. La concentración tisular mostró que $\mathrm{SeO}$ incrementa en mayor medida el Se, tanto en la sangre de la cerda (0.209 ppm; $\mathrm{p}<0.00001)$, calostro (0.324 ppm; $\mathrm{p}=0.002) \mathrm{y}$, leche $(0.145 \mathrm{ppm} ; \mathrm{p}<0.00001)$, así como en tejidos de la progenie como hígado (1.462 ppm; $\mathrm{p}=0.048)$ músculo (0.319 ppm; $\mathrm{p}<0.00001)$, riñón $(1.275 \mathrm{ppm} ; \mathrm{p}=0.004)$ y sangre $(0.18 \mathrm{ppm} ; \mathrm{p}=0.005)$. Sin embargo, la mayor concentración tisular de Se alcanzado por

${ }^{1}$ Facultad de Medicina Veterinaria y Zootecnia, Universidad Central del Ecuador, Quito, Ecuador

${ }^{2}$ Escuela de Postgrado, Universidad Nacional Agraria La Molina, Lima, Perú

${ }^{3}$ E-mail: jrquisirumbay@uce.edu.ec

Recibido: 20 de julio de 2020

Aceptado para publicación: 4 de septiembre de 2021

Publicado: 27 de octubre de 2021

(CLos autores. Este artículo es publicado por la Rev Inv Vet Perú de la Facultad de Medicina Veterinaria, Universidad Nacional Mayor de San Marcos. Este es un artículo de acceso abierto, distribuido bajo los términos de la licencia Creative Commons Atribución 4.0 Internacional (CC BY 4.0) [https:// creativecommons.org/licenses/by/4.0/deed.es] que permite el uso, distribución y reproducción en cualquier medio, siempre que la obra original sea debidamente citada de su fuente original 
$\mathrm{SeO}$ no fue suficiente para incrementar la actividad de la enzima glutatión peroxidasa (GSH-Px) en suero en cerdas ( $0.912 \mathrm{U} / \mathrm{ml} ; \mathrm{p}=0.119)$ ni en lechones $(0.286 \mathrm{U} / \mathrm{ml} ; \mathrm{p}=0.446)$. El calostro ( $408.78 \mathrm{U} / \mathrm{ml} ; \mathrm{p}=0.048)$ y leche $(239.43 \mathrm{U} / \mathrm{ml} ; \mathrm{p}<0.00001)$ presentaron una mayor actividad enzimática con $\mathrm{SeO}$. La fuente de Se suplementada en la dieta no genera diferencia en el desempeño reproductivo en cerdas. El SeO genera mayor concentración tisular en cerdas y su progenie. La actividad de GSH-Px no presenta diferencias en cerdas ni lechones, no así en calostro y leche.

Palabras clave: cerdas, lechones, selenio, reproducción, glutatión peroxidasa

\section{AbSTRACT}

The effect of selenium supplementation on reproductive and productive performance, tissue concentration and GSH-Px activity in sows and their progeny was evaluated in a meta-analysis study. Twelve articles were used that included 4698 animals. Under the random effects model, effect size, heterogeneity, meta-regressions and publication bias were determined. Reproductive variables were not affected by the source of selenium. Piglet birth weight was higher $(\mathrm{p}=0.031)$ in sows that received inorganic selenium (SeI) 1.47 vs. $1.46 \mathrm{~kg}$ from the organic source (SeO). Piglet weaning weight was $135 \mathrm{~g}$ greater $(\mathrm{p}=0.105)$ in sows from the $\mathrm{SeO}$ group. Tissue concentration showed that $\mathrm{SeO}$ increases Se to a greater extent in sow blood $(0.209 \mathrm{ppm} ; \mathrm{p}<0.00001)$, colostrum $(0.324 \mathrm{ppm} ; \mathrm{p}=0.002)$, milk $(0.145 \mathrm{ppm} ; \mathrm{p}<0.00001)$ and in tissues of progeny as liver $(1.462 \mathrm{ppm} ; \mathrm{p}=0.048)$ muscle ( $0.319 \mathrm{ppm} ; \mathrm{p}<0.00001)$, kidney ( $1.275 \mathrm{ppm} ; \mathrm{p}=0.004)$ and blood $(0.18 \mathrm{ppm} ; \mathrm{p}=0.005)$. However, the higher tissue concentration of Se reached by $\mathrm{SeO}$ was not sufficient to increase the activity of the enzyme glutathione peroxidase (GSH-Px) in serum in sows $(0.912 \mathrm{U} / \mathrm{ml} ; \mathrm{p}=0.119)$ or in piglets $(0.286 \mathrm{U} / \mathrm{ml} ; \mathrm{p}=0.446)$. Colostrum $(408.78 \mathrm{U} / \mathrm{ml} ; \mathrm{p}=0.048)$ and milk $(239.43 \mathrm{U} / \mathrm{ml} ; \mathrm{p}<0.00001)$ presented higher enzymatic activity with $\mathrm{SeO}$. The source of Se supplemented in the diet does not make a difference in reproductive performance in sows. $\mathrm{SeO}$ generates a higher tissue concentration in sows and their progeny. GSH-Px activity does not show differences in sows or piglets, but not in colostrum and milk.

Key words: sows, piglets, selenium, reproduction, glutathione peroxidase

\section{INTRODUCCIÓN}

El selenio ( $\mathrm{Se}$ ) es considerado como un elemento traza crucial en la nutrición animal (Oropeza-Moe et al., 2015). La función biológica del Se está ligada a las seleno-proteínas, un grupo de proteínas cuyo rol principal es la regulación del estrés oxidativo, fertilidad y función inmune (Labunskyy et al., 2014). La familia de las seleno-proteínas incluye al menos 25 proteínas en eucariotas y al menos 21 seleno-proteínas han sido identificadas en tejidos del cerdo (Liu et al., 2012;
Zhao et al., 2015). El Se es requerido para una adecuada función inmune y el insuficiente consumo dietario de Se puede causar severas deficiencias inmunes (McKenzie et al., 1998; Rayman, 2000) y disminuir el rendimiento reproductivo (Surai y Fisinin, 2016). Por lo tanto, una adecuada suplementación de $\mathrm{Se}$ puede ayudar a prevenir o aliviar la morbilidad y la mortalidad asociada a desbalances del sistema inmune que pueden conducir a enfermedades inflamatorias (Kalantari et al., 2008; Chen et al., 2014). La suplementación materna con Se provee protección antioxidante esencial a la descendencia (Pappas et al., 2008). 
Las cerdas están expuestas a diferentes tipos de estrés, por ejemplo, estrés social, calórico y oxidativo durante el ciclo de producción (Mendl et al., 1992; Tsuma et al., 1995; Munsterhjelm et al., 2008; BerchieriRonchi et al., 2011). El incremento en el estrés oxidativo durante la gestación y lactancia debido a un bajo consumo de compuestos con función antioxidante como el selenio puede afectar negativamente el desarrollo embrionario, el crecimiento y salud fetal, el número de mortinatos y tamaño de la camada, así como el crecimiento de los lechones (Renaudeau y Noblet, 2001; Williams et al., 2013; Zhao et al., 2013). Tamaños pequeños de camadas, incremento en la mortalidad fetal y lechones débiles han sido asociados con deficiencia de Se o vitamina $E$ en cerdas (Wuryastuti et al., 1993). El consumo adecuado de alimento en cerdas de alto rendimiento durante la lactancia es un desafío (Thingnes et al., 2015). La optimización de la composición de la dieta puede mejorar el consumo de alimento de la cerda y mejorar, por lo tanto, la composición del calostro y la leche, conduciendo a un mejor desempeño de la progenie (Krogh et al., 2015).

La composición del calostro y la leche, en cuanto a concentración de Se y de forma, es de notable importancia para los recién nacidos (Rooke y Bland, 2002; Zhan et al., 2011). Un adecuado sistema antioxidante en las cerdas ayuda a prevenir los efectos relacionados con el estrés oxidativo en la descendencia (Luo et al., 2006; Surai y Fisinin, 2016). De manera general, hay dos fuentes de Se que se emplean en la alimentación animal: selenio-orgánico $(\mathrm{SeO})$ (selenio-levadura, selenio-metionina) y selenio-inorgánico (SeI) (selenito de sodio) (National Research Council, 2012). En el selenio-levadura entre el 60 al 70\% es selenio-metionina (SeMet) y 10 al $15 \%$ es selenio-cisteína (Bierla et al., 2013).

Se ha reportado que fuentes de selenio en la dieta afectan marcadamente las concentraciones de Se en diferentes órganos y tejidos, y que la suplementación de Se orgánico incrementa de manera más efectiva la concentración de Se en calostro y leche en comparación a la forma inorgánica (Dalgaard et al., 2018), aunque también se han reportado resultados contradictorios, lo cual genera incertidumbre en los profesionales dedicados a la nutrición y alimentación de cerdos. El metaanálisis es un método estadístico que resume y cuantifica el conocimiento adquirido a través del análisis de los resultados de investigaciones ya publicados (Sauvant et al., 2008). Este procedimiento permite obtener conclusiones más sólidas y de mayor precisión que aquellas de los trabajos individuales $\mathrm{y}$, por lo tanto, una mayor potencia estadística (Catalá-López y Tobías, 2014). El objetivo de esta investigación fue determinar el efecto de la suplementación de Se (inorgánico/orgánico) sobre el desempeño reproductivo, productivo y estado antioxidante en cerdas y su progenie mediante el uso de metaanálisis.

\section{Materiales y Métodos}

Se realizó una búsqueda electrónica de artículos científicos en revistas indexadas con revisión de pares basado en la metodología de Bougouin et al. (2014). Para este efecto se consultaron las bases de datos $\mathrm{CAB}$ direct, Elsevier biobase-CABS, Google Scholar, MEDLINE, PubMed, Science Direct, Scopus, Academic Search Complete, CAB Abstract, y el Directory of Open Access Journals, utilizando un conjunto de palabras clave: selenio, Se, dieta, alimento, nutrición, orgánico, inorgánico, cerdas, glutatión peroxidasa, GSH-Px y sus equivalentes en inglés, sin restricción de fecha.

Se revisaron artículos realizados en cerdas en etapa reproductiva en los cuales el selenio fue administrado a través de la dieta y en animales sanos. Los artículos debían incluir información respecto al número de unidades experimentales por tratamiento, no menos de dos tratamientos, con fuentes de 
selenio, sea inorgánica: selenito de sodio u orgánica: selenio-levadura, suplementación de $\mathrm{Se}, \mathrm{Zn}, \mathrm{Cu}$, vitamina A y E nutrientes en premezclas comerciales. Solo se incluyeron niveles de 3 ppm o inferiores de selenio suplementados en la dieta para evitar efectos nocivos en el consumo o interacción con otros minerales en la dieta (National Research Council, 2012; PIC, 2016; Rostagno et al., 2017). Adicionalmente, los artículos debían incluir los valores de la media (promedio) y alguna medida de variación de la variable en estudio.

Los datos fueron analizados con el programa MIX 2.0 Pro en Microsoft Excel (Bax, 2016). Se determinó el tamaño del efecto global de la suplementación de selenio sobre el número de lechones nacidos totales, nacidos vivos, nacidos muertos, lechones destetados, peso promedio del lechón al nacimiento, al destete, concentración de Se en sangre (cerda y lechones), calostro y leche, y actividad de la enzima glutatión peroxidasa en suero (cerdas y lechones), calostro y leche; concentración de Se en hígado, músculo y riñón de lechones por diferencia de medias (DM), con intervalos de confianza al $95 \%$.

La heterogeneidad se evaluó por medio del índice de inconsistencia $\left(\mathrm{I}^{2}\right)$ (Higgins y Thompson 2002). El sesgo de publicación se evaluó mediante la prueba de regresión de Egger (Egger et al., 1997). Se utilizó un modelo de efectos aleatorios según las recomendaciones de Borenstein et al. (2011) y Sauvant et al. (2008). Se ejecutaron 17 metaanálisis que incluyó un total de 204 registros de comparación y un $n=4698$, a partir de 12 artículos científicos: Mahan y Kim (1996), Mahan (2000), Kim y Mahan (2001a), a ara et al. (2005), Yoon y McMillan (2006), Quesnel et al. (2008), Svoboda et al. (2008), Hu et al. (2011), Zhan et al. (2011), Ma et al. (2014), Chen et al. (2016a, 2016b).

Para los cálculos correspondientes, el grupo tratamiento consistió en selenio orgánico (selenio-levadura) y el grupo control en selenio inorgánico (selenito de sodio). Las razas involucradas en el metaanálisis fueron Landrace, Large White, Yorkshire e híbridos Yorkshire-Landrace y Duroc-Hampshire x Yorkshire-Landrace. La heterogeneidad entre estudios se determinó mediante meta-regresiones utilizando como covariables: número de unidades experimentales por tratamiento, nivel de suplementación en la dieta de selenio, cobre, zinc, vitaminas A y E. No se consideraron como covariables al número de animales por unidad experimental ni el número de animales muestreados por unidad experimental pues para todos los estudios utilizados el valor fue de 1 .

\section{Resultados}

La suplementación dietética de selenio orgánico en cerdas presenta una tendencia a mejorar el rendimiento reproductivo (Cuadro 1). Sin embargo, la diferencia no fue significativa: número de lechones nacidos totales $(\mathrm{p}=0.869)$, nacidos vivos $(\mathrm{p}=0.758)$, nacidos muertos $(\mathrm{p}=0.426)$. De otra parte, se aprecia una mejora evidente en el peso promedio del lechón al nacimiento $(\mathrm{p}=0.031)$ en cerdas que recibieron selenio inorgánico que en aquellas que recibieron Se orgánico (1.469 vs. 1.455 $\mathrm{kg})$. No hubo diferencia significativa en el peso del lechón al destete $(\mathrm{p}=0.105)$, pero los lechones de la fuente orgánica presentaron pesos ligeramente superiores $(5.353 \mathrm{~kg})$ frente a los lechones destetados de la fuente inorgánica $(5.266 \mathrm{~kg})$. La suplementación de $\mathrm{SeO}$ determina una mayor concentración de Se en sangre en las cerdas $(p<0.00001)$ en comparación a la fuente inorgánica; sin embargo, no se encontró diferencia en la actividad de GSH-Px (medida en suero) entre ambas fuentes $(\mathrm{p}=0.119)$.

La concentración de Se en calostro fue de aproximadamente el doble $(\mathrm{p}=0.002)$ en las cerdas que se alimentaron de $\mathrm{SeO}(0.324$ ppm) en comparación con la fuente inorgánica $(0.167 \mathrm{ppm})$. Una tendencia similar presentó la concentración de $\mathrm{Se}$ en leche donde $\mathrm{SeO}$ 
Cuadro 1. Resumen de la variable respuesta y tamaño de efecto en cerdas y su progenie al suplementar selenio orgánico (selenio-levadura) a los grupos tratados y selenio inorgánico (selenito de sodio) a los grupos control en un estudio de metaanálisis

\begin{tabular}{|c|c|c|c|c|c|c|c|c|}
\hline \multirow{3}{*}{ Metaanálisis } & \multicolumn{8}{|c|}{ Resumen variable respuesta y tamaño de efecto } \\
\hline & \multicolumn{2}{|c|}{ Tratamiento } & \multicolumn{2}{|c|}{ Control } & \multicolumn{4}{|c|}{ Tamaño de efecto } \\
\hline & Media & SD & Media & SD & $\mathrm{DM}$ & IC- & IC+ & $\mathrm{p}$ \\
\hline $\begin{array}{l}\text { Nacidos totales } \\
\text { por cerda }(n)\end{array}$ & 11.86 & 1.11 & 11.75 & 1.88 & 0.078 & -0.850 & 1.006 & 0.869 \\
\hline $\begin{array}{l}\text { Nacidos vivos por } \\
\text { cerda (n) }\end{array}$ & 11.13 & 1.08 & 11.04 & 1.60 & 0.130 & -0.700 & 0.961 & 0.758 \\
\hline $\begin{array}{l}\text { Nacidos muertos } \\
\text { por cerda }(\mathrm{n})\end{array}$ & 0.88 & 0.51 & 0.75 & 0.56 & 0.111 & -0.163 & 0.386 & 0.426 \\
\hline $\begin{array}{l}\text { Destetados por } \\
\text { cerda }(\mathrm{n})\end{array}$ & 9.72 & 1.01 & 9.38 & 0.99 & 0.250 & -0.217 & 0.717 & 0.294 \\
\hline $\begin{array}{l}\text { Peso al nacer } \\
(\mathrm{kg})\end{array}$ & 1.46 & 0.08 & 1.47 & 0.11 & -0.038 & -0.073 & -0.004 & 0.031 \\
\hline $\begin{array}{l}\text { Peso al destete } \\
(\mathrm{kg})\end{array}$ & 5.35 & 0.99 & 5.27 & 1.01 & 0.135 & -0.028 & 0.299 & 0.105 \\
\hline $\begin{array}{l}\text { Se en sangre cerda } \\
(\mathrm{ppm})\end{array}$ & 0.209 & 0.070 & 0.172 & 0.015 & 0.020 & 0.014 & 0.027 & $<0.00001$ \\
\hline $\begin{array}{l}\text { GSH-Px suero } \\
\text { cerda } / \mathrm{U} / \mathrm{ml} \text { ) }\end{array}$ & 0.912 & 0.318 & 0.968 & 0.369 & -0.050 & -0.113 & 0.013 & 0.119 \\
\hline $\begin{array}{l}\text { Se en calostro } \\
(\mathrm{ppm})\end{array}$ & 0.324 & 0.357 & 0.167 & 0.049 & 0.041 & 0.016 & 0.067 & 0.002 \\
\hline $\begin{array}{l}\text { GSH-Px en } \\
\text { calostro (U/ml) }\end{array}$ & 408.78 & 170.53 & 347.93 & 134.34 & 57.50 & 0.608 & 114.384 & 0.048 \\
\hline $\begin{array}{l}\text { Se en leche } \\
(\mathrm{ppm})\end{array}$ & 0.145 & 0.165 & 0.058 & 0.041 & 0.034 & 0.020 & 0.047 & $<0.00001$ \\
\hline $\begin{array}{l}\text { GSH-Px en leche } \\
(\mathrm{U} / \mathrm{ml})\end{array}$ & 239.438 & 93.530 & 214.155 & 75.530 & 17.885 & 10.361 & 25.410 & $<0.00001$ \\
\hline $\begin{array}{l}\text { Se en hígado } \\
\text { lechón (ppm) }\end{array}$ & 1.462 & 2.815 & 0.284 & 0.142 & 0.071 & 0.001 & 0.142 & 0.048 \\
\hline $\begin{array}{l}\text { Se en músculo } \\
\text { lechón (ppm) }\end{array}$ & 0.319 & 0.586 & 0.071 & 0.045 & 0.042 & 0.024 & 0.059 & $<0.00001$ \\
\hline $\begin{array}{l}\text { Se riñón lechón } \\
\text { (ppm) }\end{array}$ & 1.275 & 0.752 & 0.800 & 0.601 & 0.181 & 0.057 & 0.306 & 0.004 \\
\hline $\begin{array}{l}\text { Se en sangre } \\
\text { lechón (ppm) }\end{array}$ & 0.180 & 0.176 & 0.099 & 0.120 & 0.018 & 0.005 & 0.031 & 0.005 \\
\hline $\begin{array}{l}\text { GSH-Px suero } \\
\text { lechón }(\mathrm{U} / \mathrm{ml})\end{array}$ & 0.286 & 0.155 & 0.268 & 0.153 & 0.013 & -0.020 & 0.046 & 0.446 \\
\hline
\end{tabular}

$\mathrm{IC}=$ intervalo de confianza al 95\%; $\mathrm{p}=$ valor de probabilidad 
Cuadro 2. Índice de inconsistencia (heterogeneidad) en variables evaluadas en cerdas y su progenie al suplementar selenio orgánico (selenio-levadura) a los grupos tratados y selenio inorgánico (selenito de sodio) a los grupos control en un estudio de metaanálisis

\begin{tabular}{lc}
\hline Metaanálisis & $\mathrm{I}^{2}(\%)$ \\
\hline Nacidos totales / cerda (n) & 82.58 \\
Nacidos vivos / cerda (n) & 83.50 \\
Nacidos muertos / cerda (n) & 75.53 \\
Destetados por cerda (n) & 63.10 \\
Peso al nacer (kg) & 0.00 \\
Peso al destete (kg) & 61.26 \\
Se en sangre cerda (ppm) & 53.41 \\
GSH-Px suero cerda /U/ml) & 2.29 \\
Se en calostro (ppm) & 79.96 \\
GSH-Px en calostro (U/ml) & 86.55 \\
Se en leche (ppm) & 96.35 \\
GSH-Px en leche (U/ml) & 58.08 \\
Se en hígado lechón (ppm) & 92.13 \\
Se en músculo lechón (ppm) & 83.97 \\
Se riñón lechón (ppm) & 82.81 \\
Se en sangre lechón (ppm) & 86.30 \\
GSH-Px suero lechón (U/ml) & 17.38 \\
\hline
\end{tabular}

triplicó $(\mathrm{p}<0.00001)$ la concentración $(0.145$ ppm) vs. SeI (0.058 ppm). Respecto a la actividad de GSH-Px, se encontró que el $\mathrm{SeO}$ versus SeI provoca un incremento significativo $(\mathrm{p}<0.05)$ en la actividad de esta enzima, tanto a nivel de calostro (408.78 vs. 347.93 $\mathrm{U} / \mathrm{ml}$ ) como en la leche (239.43 vs. 214.15 $\mathrm{U} / \mathrm{ml}$ ). Los lechones provenientes de las cerdas que recibieron $\mathrm{SeO}$ concentraron en mayor medida $(p<0.05)$ el Se en los diferentes órganos medidos en comparación con el Se I: hígado 1.462 vs. 0.284 ppm; músculo 0.319 vs. 0.071 ppm; riñón 1.275 vs 0.8 ppm y sangre 0.18 vs $0.099 \mathrm{ppm}$. La actividad de la enzima GSH-Px medida en suero en lechones presentó el mismo comportamiento que en sus madres; esto es, no se encontró diferencia $(\mathrm{p}=0.446)$ entre la fuente orgánica $(0.286$ $\mathrm{U} / \mathrm{ml})$ frente a la inorgánica $(0.268 \mathrm{U} / \mathrm{ml})$.

La heterogeneidad de los resultados en las variables en las cerdas y su progenie por efecto de la suplementación con selenio en el metaanálisis se presenta en el Cuadro 2. El análisis de meta-regresión determinó que el número de repeticiones afecta de manera significativa $(p<0.05)$ cada una de las variables evaluadas, excepto al peso promedio del lechón al nacimiento $(\mathrm{p}=0.6853)$, concentración de Se en hígado $(\mathrm{p}=0.716)$, músculo $(\mathrm{p}=0.796)$ $y$ actividad de GSH-Px en suero $(\mathrm{p}=0.582)$ medidas en lechón (Cuadro 3). El nivel de selenio suplementando a través de la dieta tiene un efecto significativo sobre peso promedio del lechón al destete, concentración de Se en sangre (cerda), concentración de Se en calostro y leche, concentración de Se en hígado, músculo y riñón en lechones (Cuadro 3). Adicionalmente, se aprecia que los niveles de otros nutrientes como $\mathrm{Zn}, \mathrm{Cu}$, vitaminas A y E afectan algunas de las variables evaluadas (Cuadros 4 y 5).

\section{Discusión}

En el presente trabajo se puede apreciar que no existe diferencia en las variables reproductivas para cualquiera de las fuentes de selenio analizadas, excepto para la variable peso promedio del lechón al nacimiento, que resultó más alta para $\operatorname{SeI}(\mathrm{p}=0.031)$. Los lechones del grupo SeI pesan $38.39 \mathrm{~g}$ más en comparación con los lechones procedentes de cerdas que recibieron $\mathrm{SeO}$. Sin embargo, al destete los lechones $\mathrm{SeO}$ presentaron mayor peso en comparación con los lechones SeI, 5.35 vs. $5.27 \mathrm{~kg}$ respectivamente. Si bien la diferencia no fue significativa en el peso al destete $(\mathrm{p}=0.104)$, los lechones del grupo $\mathrm{SeO}$ pesaron $135.26 \mathrm{~g}$ adicionales, lo cual está asociado a una mejora en la actividad de las enzimas pancreáticas (amilasa, lipasa y proteasa) y del estado de la glándula tiroides como lo demuestra el trabajo realizado por 
Cuadro 3. Meta-regresión del número de repeticiones y nivel de Se sobre la respuesta productiva de cerdos y lechones

\begin{tabular}{|c|c|c|c|c|c|c|c|c|}
\hline \multirow{3}{*}{ Metaanálisis } & \multicolumn{4}{|c|}{ Número de repeticiones } & \multicolumn{4}{|c|}{ Nivel de Se } \\
\hline & \multicolumn{2}{|c|}{ Intercepto } & \multicolumn{2}{|c|}{ Coef. Regresión } & \multicolumn{2}{|c|}{ Intercepto } & \multicolumn{2}{|c|}{ Coef. Regresión } \\
\hline & Estimado & $\mathrm{p}$ & Estimado & $\mathrm{p}$ & Estimado & $\mathrm{p}$ & Estimado & $\mathrm{p}$ \\
\hline $\begin{array}{l}\text { Nacidos totales / } \\
\text { cerda (n) }\end{array}$ & -1.202 & $<0.001$ & 0.042 & $<0.001$ & 0.057 & 0.801 & 0.107 & 0.823 \\
\hline $\begin{array}{l}\text { Nacidos vivos / } \\
\text { cerda (n) }\end{array}$ & -0.552 & 0.052 & 0.028 & 0.001 & 0.164 & 0.376 & 0.183 & 0.532 \\
\hline $\begin{array}{l}\text { Nacido muerto / } \\
\text { cerda (n) }\end{array}$ & 0.375 & $<0.001$ & -0.011 & 0.006 & 0.322 & 0.002 & -0.623 & 0.080 \\
\hline $\begin{array}{l}\text { Destetados por } \\
\text { cerda }(n)\end{array}$ & -0.366 & 0.157 & 0.021 & 0.004 & 0.386 & 0.049 & -0.231 & 0.658 \\
\hline $\begin{array}{l}\text { Peso al nacer } \\
\text { (kg) }\end{array}$ & -0.020 & 0.689 & -0.002 & 0.685 & -0.044 & 0.050 & 0.016 & 0.668 \\
\hline $\begin{array}{l}\text { Peso al destete } \\
(\mathrm{kg})\end{array}$ & 0.316 & $<0.001$ & -0.009 & $<0.001$ & 0.046 & 0.444 & 0.319 & 0.012 \\
\hline $\begin{array}{l}\text { Se en sangre } \\
\text { cerda }(\mathrm{ppm})\end{array}$ & 0.008 & 0.037 & 0.000 & $<0.001$ & -0.005 & 0.396 & 0.104 & $<0.001$ \\
\hline $\begin{array}{l}\text { GSH-Px suero } \\
\text { cerda /U/ml) }\end{array}$ & 0.061 & 0.245 & -0.019 & 0.008 & -0.074 & 0.045 & 0.049 & 0.202 \\
\hline $\begin{array}{l}\text { Se en calostro } \\
(\mathrm{ppm})\end{array}$ & 0.018 & 0.004 & 0.001 & 0.005 & -0.027 & 0.231 & 0.198 & 0.010 \\
\hline $\begin{array}{l}\text { GSH-Px en } \\
\text { calostro (U/ml) }\end{array}$ & 11.479 & 0.074 & 1.658 & $<0.001$ & & ivel únic & $0.3 \mathrm{ppm}$ & \\
\hline $\begin{array}{l}\text { Se en leche } \\
(\mathrm{ppm})\end{array}$ & 0.055 & $<0.001$ & -0.001 & $<0.001$ & 0.019 & $<0.001$ & 0.103 & $<0.001$ \\
\hline $\begin{array}{l}\text { GSH-Px en } \\
\text { leche (U/ml) }\end{array}$ & 9.988 & 0.001 & 0.853 & 0.006 & & ivel únic & $0.3 \mathrm{ppm}$ & \\
\hline $\begin{array}{l}\text { Se en hígado } \\
\text { lechón (ppm) }\end{array}$ & 0.102 & 0.126 & -0.004 & 0.716 & -0.020 & 0.667 & 0.343 & 0.034 \\
\hline $\begin{array}{l}\text { Se en músculo } \\
\text { lechón (ppm) }\end{array}$ & 0.026 & 0.051 & 0.001 & 0.796 & 0.003 & 0.693 & 0.097 & $<0.001$ \\
\hline $\begin{array}{l}\text { Se riñón lechón } \\
\text { (ppm) }\end{array}$ & 1.188 & $<0.001$ & -0.177 & $<0.001$ & -0.015 & 0.717 & 0.485 & $<0.001$ \\
\hline $\begin{array}{l}\text { Se en sangre } \\
\text { lechón (ppm) }\end{array}$ & -0.034 & $<0.001$ & 0.006 & $<0.001$ & 0.005 & 0.499 & -0.004 & 0.869 \\
\hline $\begin{array}{l}\text { GSH-Px suero } \\
\text { lechón }(\mathrm{U} / \mathrm{ml})\end{array}$ & 0.021 & 0.398 & -0.003 & 0.582 & -0.009 & 0.610 & 0.034 & 0.057 \\
\hline
\end{tabular}

$\mathrm{p}=$ valor de probabilidad 
Cuadro 4. Meta-regresión del nivel de $\mathrm{Zn}$ y $\mathrm{Cu}$ sobre la respuesta productiva de cerdos y lechones que recibieron dietas suplementadas con selenio

\begin{tabular}{|c|c|c|c|c|c|c|c|c|}
\hline \multirow{3}{*}{ Metaanálisis } & \multicolumn{4}{|c|}{ Nivel de Zn } & \multicolumn{4}{|c|}{ Nivel de $\mathrm{Cu}$} \\
\hline & \multicolumn{2}{|c|}{ Intercepto } & \multicolumn{2}{|c|}{ Coef. Regresión } & \multicolumn{2}{|c|}{ Intercepto } & \multicolumn{2}{|c|}{ Coef. Regresión } \\
\hline & Estimado & $\mathrm{p}$ & Estimado & $\mathrm{p}$ & $\begin{array}{c}\text { Estimad } \\
0\end{array}$ & $\mathrm{p}$ & Estimado & $\mathrm{p}$ \\
\hline $\begin{array}{l}\text { Nacidos totales } \\
\text { / cerda (n) }\end{array}$ & 1.738 & $<0.001$ & -0.024 & $<0.001$ & 1.758 & $<0.001$ & -0.246 & $<0.001$ \\
\hline $\begin{array}{l}\text { Nacidos vivos / } \\
\text { cerda (n) }\end{array}$ & 1.288 & $<0.001$ & -0.014 & 0.004 & 1.385 & $<0.001$ & -0.161 & $<0.001$ \\
\hline $\begin{array}{l}\text { Nacido muerto } \\
\text { / cerda (n) }\end{array}$ & -0.403 & 0.038 & 0.006 & 0.002 & -0.024 & 0.926 & 0.026 & 0.442 \\
\hline $\begin{array}{l}\text { Destetados por } \\
\text { cerda (n) }\end{array}$ & 1.062 & $<0.001$ & -0.011 & 0.005 & 1.059 & $<0.001$ & -0.113 & 0.003 \\
\hline $\begin{array}{l}\text { Peso al nacer } \\
(\mathrm{kg})\end{array}$ & -0.075 & 0.622 & 0.000 & 0.809 & 0.068 & 0.430 & -0.011 & 0.207 \\
\hline $\begin{array}{l}\text { Peso al destete } \\
(\mathrm{kg})\end{array}$ & -0.263 & 0.077 & 0.005 & 0.003 & -0.147 & 0.258 & 0.035 & 0.014 \\
\hline $\begin{array}{l}\text { Se en sangre } \\
\text { cerda (ppm) }\end{array}$ & 0.041 & $<0.001$ & 0.000 & $<0.001$ & 0.058 & $<0.001$ & -0.006 & $<0.001$ \\
\hline $\begin{array}{l}\text { GSH-Px suero } \\
\text { cerda } / \mathrm{U} / \mathrm{ml} \text { ) }\end{array}$ & \multicolumn{4}{|c|}{ Nivel único 120 ppm } & -0.469 & 0.121 & 0.049 & 0.163 \\
\hline $\begin{array}{l}\text { Se en calostro } \\
(\mathrm{ppm})\end{array}$ & 0.026 & 0.010 & 0.000 & 0.716 & 0.013 & 0.107 & 0.003 & 0.019 \\
\hline $\begin{array}{l}\text { GSH-Px en } \\
\text { calostro (U/ml) }\end{array}$ & \multicolumn{4}{|c|}{ Nivel único 50 ppm } & \multicolumn{4}{|c|}{ Nivel único 5 ppm } \\
\hline $\begin{array}{l}\text { Se en leche } \\
(\mathrm{ppm})\end{array}$ & -0.008 & 0.065 & 0.001 & $<0.001$ & -0.013 & 0.002 & 0.007 & $<0.001$ \\
\hline $\begin{array}{l}\text { GSH-Px en } \\
\text { leche }(\mathrm{U} / \mathrm{ml})\end{array}$ & \multicolumn{4}{|c|}{ Nivel único 50 ppm } & \multicolumn{4}{|c|}{ Nivel único 5 ppm } \\
\hline $\begin{array}{l}\text { Se en hígado } \\
\text { lechón (ppm) }\end{array}$ & 0.086 & $<0.001$ & 0.000 & 0.713 & 0.093 & 0.034 & -0.003 & 0.725 \\
\hline $\begin{array}{l}\text { Se en músculo } \\
\text { lechón (ppm) }\end{array}$ & 0.011 & 0.088 & 0.000 & 0.003 & -0.007 & 0.541 & 0.006 & 0.002 \\
\hline $\begin{array}{l}\text { Se riñón lechón } \\
\text { (ppm) }\end{array}$ & -0.264 & 0.045 & 0.008 & 0.002 & -0.422 & 0.021 & 0.110 & 0.002 \\
\hline $\begin{array}{l}\text { Se en sangre } \\
\text { lechón (ppm) }\end{array}$ & -0.020 & $<0.001$ & 0.000 & $<0.001$ & -0.048 & $<0.001$ & 0.010 & $<0.001$ \\
\hline $\begin{array}{l}\text { GSH-Px suero } \\
\text { lechón }(\mathrm{U} / \mathrm{ml})\end{array}$ & 0.195 & 0.706 & -0.002 & 0.721 & 0.032 & 0.633 & -0.002 & 0.738 \\
\hline
\end{tabular}

$\mathrm{p}=$ valor de probabilidad 
Cuadro 5. Meta-regresión del nivel de vitaminas A y E sobre la respuesta productiva de cerdos y lechones que recibieron dietas suplementadas con selenio

\begin{tabular}{|c|c|c|c|c|c|c|c|c|}
\hline \multirow{3}{*}{ Metaanálisis } & \multicolumn{4}{|c|}{ Nivel de vitamina A } & \multicolumn{4}{|c|}{ Nivel de vitamina $E$} \\
\hline & \multicolumn{2}{|c|}{ Intercepto } & \multicolumn{2}{|c|}{ Coef. Regresión } & \multicolumn{2}{|c|}{ Intercepto } & \multicolumn{2}{|c|}{ Coef. Regresión } \\
\hline & Estimado & $\mathrm{p}$ & Estimado & $\mathrm{p}$ & Estimado & $\mathrm{p}$ & Estimado & $\mathrm{p}$ \\
\hline $\begin{array}{l}\text { Nacidos totales / } \\
\text { cerda (n) }\end{array}$ & -0.23594 & 0.697 & 0.00003 & 0.572 & 0.83725 & 0.026 & -0.01295 & 0.029 \\
\hline $\begin{array}{l}\text { Nacidos vivos / } \\
\text { cerda (n) }\end{array}$ & 0.28176 & 0.501 & 0.00000 & 0.906 & 1.32336 & $<0.001$ & -0.02015 & $<0.001$ \\
\hline $\begin{array}{l}\text { Nacido muerto / } \\
\text { cerda (n) }\end{array}$ & 0.56122 & $<0.001$ & -0.00006 & $<0.001$ & 0.24850 & 0.103 & -0.00164 & 0.580 \\
\hline $\begin{array}{l}\text { Destetados por } \\
\text { cerda (n) }\end{array}$ & -0.26673 & 0.528 & 0.00005 & 0.155 & 0.46750 & 0.074 & -0.00263 & 0.519 \\
\hline $\begin{array}{l}\text { Peso al nacer } \\
(\mathrm{kg})\end{array}$ & 0.01747 & 0.734 & -0.00001 & 0.247 & 0.00981 & 0.866 & -0.00085 & 0.384 \\
\hline $\begin{array}{l}\text { Peso al destete } \\
(\mathrm{kg})\end{array}$ & 0.45422 & $<0.001$ & -0.00003 & 0.005 & 0.24825 & 0.023 & -0.00187 & 0.354 \\
\hline $\begin{array}{l}\text { Se en sangre } \\
\text { cerda (ppm) }\end{array}$ & 0.00400 & 0.402 & 0.00000 & $<0.001$ & 0.02237 & $<0.001$ & 0.00000 & 0.995 \\
\hline $\begin{array}{l}\text { GSH-Px suero } \\
\text { cerda } / \mathrm{U} / \mathrm{ml} \text { ) }\end{array}$ & 0.09597 & 0.380 & -0.00004 & 0.163 & 0.05698 & 0.492 & -0.00337 & 0.163 \\
\hline $\begin{array}{l}\text { Se en calostro } \\
(\mathrm{ppm})\end{array}$ & -0.01282 & 0.394 & 0.00000 & 0.003 & 0.01860 & 0.026 & 0.00033 & 0.115 \\
\hline $\begin{array}{l}\text { GSH-Px en } \\
\text { calostro (U/ml) }\end{array}$ & -174.965 & 0.005 & 0.01965 & 0.001 & -10.6895 & 0.314 & 1.44732 & $<0.001$ \\
\hline $\begin{array}{l}\text { Se en leche } \\
(\mathrm{ppm})\end{array}$ & 0.07026 & $<0.001$ & 0.00000 & $<0.001$ & 0.03943 & $<0.001$ & 0.00011 & 0.228 \\
\hline $\begin{array}{l}\text { GSH-Px en } \\
\text { leche }(\mathrm{U} / \mathrm{ml})\end{array}$ & -91.6944 & 0.016 & 0.01068 & 0.004 & 10.28555 & 0.084 & 0.25020 & 0.281 \\
\hline $\begin{array}{l}\text { Se en hígado } \\
\text { lechón (ppm) }\end{array}$ & 0.19731 & $<0.001$ & -0.00001 & $<0.001$ & 0.11352 & $<0.001$ & -0.00071 & 0.010 \\
\hline $\begin{array}{l}\text { Se en músculo } \\
\text { lechón (ppm) }\end{array}$ & 0.05482 & $<0.001$ & 0.00000 & $<0.001$ & 0.04767 & $<0.001$ & -0.00037 & $<0.001$ \\
\hline $\begin{array}{l}\text { Se riñón lechón } \\
\text { (ppm) }\end{array}$ & 0.49716 & $<0.001$ & -0.00003 & 0.003 & 0.09836 & 0.004 & 0.00088 & 0.186 \\
\hline $\begin{array}{l}\text { Se en sangre } \\
\text { lechón (ppm) }\end{array}$ & 0.04724 & $<0.001$ & 0.00000 & $<0.001$ & 0.01601 & $<0.001$ & -0.00021 & $<0.001$ \\
\hline $\begin{array}{l}\text { GSH-Px suero } \\
\text { lechón }(\mathrm{U} / \mathrm{ml})\end{array}$ & 0.04014 & 0.190 & -0.00001 & 0.263 & 0.06302 & 0.090 & -0.00170 & 0.120 \\
\hline
\end{tabular}

$\mathrm{p}=$ valor de probabilidad 
Zhan et al. (2011). Además, hay un mejor estado inmunitario generado por la forma orgánica (Dalgaard et al., 2018), de esta manera el lechón destina los nutrientes ingeridos a la ganancia de peso y no hacia reacciones catabólicas.

De los metaanálisis realizados en este trabajo de investigación se puede apreciar claramente como la suplementación de selenio orgánico permite concentrar mayor cantidad de este micromineral en el suero de la cerda $(\mathrm{DM}=0.020 ; \mathrm{p}<0.00001)$, su calostro $(\mathrm{DM}=0.041 \mathrm{ppm} ; \mathrm{p}=0.00161)$ y leche ( $\mathrm{DM}=0.033$ ppm; $\mathrm{p}<0.00001)$. Lo anterior está relacionado a que el $\mathrm{SeO}$ se absorbe en mayor medida (70-90\%) en comparación al SeI $(<60 \%)$ (Arnér, 2010) y a que en la cerda el $\mathrm{SeO}$ forma reservas corporales de $\mathrm{Se} \mathrm{a}$ nivel muscular en forma de selenio-metionina y selenio-cisteína que son liberados durante etapas de mayor exigencia metabólica como la etapa final de la gestación y la lactancia (Dalgaard et al., 2018), incrementando de esta manera el nivel de Se en calostro y leche. Estudios realizados en vacas lecheras muestran que la suplementación de $\mathrm{SeO}$ también incrementa la concentración de Se en leche, principalmente en la proteína en forma de SeMet (Phipps et al., 2008).

En lechones se aprecia que aquellos que se alimentaron del calostro y leche provenientes de cerdas que recibieron $\mathrm{SeO}$, concentraron en mayor medida este mineral traza. Es así que, hígado ( $\mathrm{DM}=0.071 \mathrm{ppm} ; \mathrm{p}=0.048)$, músculo ( $\mathrm{DM}=0.041 \mathrm{ppm}$; $\mathrm{p}<0.00001)$, riñón $(\mathrm{DM}=0.181 \mathrm{ppm} ; \mathrm{p}=0.004)$ y sangre $(\mathrm{DM}=0.018 \mathrm{ppm} ; \mathrm{p}=0.004)$ presentaron diferencias significativas frente a los lechones provenientes de cerdas alimentadas con SeI. La mayor concentración de Se encontrada en los órganos en los lechones $(\mathrm{SeO})$ está asociado a que ingieren mayor cantidad de selenio a través del calostro y leche, además de recibirlo en una forma también orgánica selenio-metionina y selenio-cisteína. De otra parte, en los estudios que se midió la concentración de Se, se aprecia que el efecto entre estudios fue inconsistente $\left(\mathrm{I}^{2}>50 \%\right)$ (Cuadro 2). Los análisis de meta-regresión determinaron que el nivel de Se en la dieta tiene efecto significativo sobre la concentración de Se medida en los diferentes tejidos, siendo que a medida que se incrementa el nivel de Se en la dieta aumenta la concentración de Se tisular (Cuadro 3).

La actividad de la enzima GSH-Px medida en el suero de las cerdas fue ligeramente superior en aquellas alimentadas a partir de la forma inorgánica $0.968 \mathrm{U} / \mathrm{ml}$ frente a la forma orgánica $0.912 \mathrm{U} / \mathrm{ml}$; sin embargo, esta diferencia no fue significativa $(\mathrm{p}=0.119)$. Esta respuesta también se ha observado en cerdos en etapas de crecimiento y engorde en donde aquellos que recibieron selenito de sodio presentaron una mayor actividad de esta enzima (Kim y Mahan, 2001b). De manera similar se encontró que pollos de engorde que recibieron selenito de sodio presentaron mayor producción y actividad de GSH-Px al compararlo frente a $\mathrm{SeO}$ (Ahmad et al., 2012). Así se demuestra que no existe una relación entre el nivel de Se en sangre con la actividad de la enzima GSH-Px medida en suero, tal y como lo demuestra el metaanálisis realizado en pollos de engorde por Zoidis et al. (2014). A nivel de calostro y leche se determinó que la suplementación de $\mathrm{SeO}$ genera una mayor actividad de la enzima GSHPx vs. la fuente inorgánica $(\mathrm{p}<0.05)$; sin embargo, en lechones la actividad de esta enzima presenta valores similares entre aquellos alimentados por cerdas que recibieron $\mathrm{SeO}$ y aquellas que recibieron $\mathrm{SeI}(\mathrm{DM}=0.01299$; $\mathrm{p}=0.445$ ), siendo además un efecto consistente como lo demuestra su baja heterogeneidad $\left(\mathrm{I}^{2}=17.38 \%\right)$. De esta manera, también se puede apreciar que a pesar de que los lechones $(\mathrm{SeO})$ que mantenían niveles altos de Se en sangre $(\mathrm{p}<0.05)$ frente a los lechones (SeI) no presentaron mayor actividad de la enzima GSH-Px. Esto puede indicar que la medición de GSH-Px no es el mejor indicador del nivel de ingesta de Se a través de la dieta. 
Desde el punto de vista económico resulta más barato la inclusión de SeI, por lo que las dietas podrían incluir únicamente la forma inorgánica para las etapas de producción recría, crecimiento y finalización (engorde). Sin embargo, la forma inorgánica de $\mathrm{Se}$ tiene algunas desventajas, que se relacionan con una interacción con otros minerales, una toxicidad relativamente alta, una baja eficiencia de transferencia a la leche, huevos y la incapacidad de construir y mantener las reservas de Se en el cuerpo (Surai, 2006; Surai y Fisinin, 2014). En cerdas se recomienda suplementar $\mathrm{SeO}$ debido a que el selenio orgánico permite acumular reservas de $\mathrm{Se}$ en tejidos, principalmente en músculos, en forma de SeMet que se puede usar en condiciones de estrés para mejorar las defensas antioxidantes (Surai, 2006; Surai y Fisinin 2014, 2015); además, el Se orgánico en forma de SeMet proporciona un transporte efectivo de Se de la cerda al feto y lechón recién nacidos a través de placenta, calostro y leche (Surai 2006; Close et al., 2008). Adicionalmente, en humanos se ha demostrado que la retención de SeMet fue de 363 días comparado frente a los 147 días para el selenito de sodio, indicando una eficiente reutilización de la SeMet (Duntas y Benvenga, 2015). Esto es importante puesto que las reservas de tejido de selenio de la cerda se agotan con el avance de la paridad, las reservas de tejidos no se movilizan fácilmente, y el tejido mamario de las hembras más viejas no puede incorporar ni transferir selenio inorgánico dietético o reservas de Se en tejidos a la leche tan eficazmente como las cerdas jóvenes (Mahan y Peters, 2004).

Otros micro-nutrientes como el $\mathrm{Zn}, \mathrm{Cu}$ (Collins, 2016; Ighodaro y Akinloye, 2018), vitaminas A (Chew, 1996) y E (Wang et al., 2017) también forman parte del sistema de defensa antioxidante del organismo. En el Cuadro 4 se aprecia que los niveles de $\mathrm{Zn}$ y $\mathrm{Cu}$ no tienen efecto significativo sobre la actividad de la enzima GSH-Px en ninguno de los tejidos medidos. Por su parte, los niveles de las vitaminas A y E tienen efecto significativo cuando la actividad fue medida en calostro y leche (Cuadro 5). Urso et al. (2015) encontraron que la vitamina $\mathrm{E}$ en pollos favorece la producción de GSH-Px; asimismo, en tanto que Aaron y Hays (2004) resaltan la importancia de considerar el número de repeticiones a fin de poder detectar diferencias significativas en caso existan. Precisamente a través del estudio de meta-regresión en este estudio se determinó que el número de repeticiones por tratamiento afectaron de manera significativa $(\mathrm{p}<0.05) 13$ de las 17 variables estudiadas (Cuadro 3). Finalmente, no se encontró sesgo de publicación en 16 de las 17 variables estudiadas, siendo la única variable que presentó sesgo fue el peso promedio del lechón al nacimiento.

\section{Conclusiones}

- Las variables reproductivas en cerdas no se ven afectadas por la fuente de $\mathrm{Se}$ suplementada a través de la dieta (orgánico/inorgánico).

- $\quad$ El SeO permite concentrar en mayor medida al Se tanto en la cerda (sangre), calostro, leche y tejidos en su progenie (hígado, músculo, riñón y sangre).

- La mayor concentración tisular de $\mathrm{Se}$ alcanzado por la fuente orgánica no fue suficiente para incrementar la actividad de la enzima GSH-Px (suero) en cerdas ni en lechones, no así en calostro y leche.

\section{Literatura Citada}

1. Aaron DK, Hays VW. 2004. How many pigs? statistical power considerations in swine nutrition experiments. J Anim Sci 82: E245-E254. doi: 10.2527/2004.8213 supplE245x

2. Ahmad H, Tian J, Wang J, Khan MA, Wang Y, Zhang L, Wang T. 2012. Effects of dietary sodium selenite and selenium yeast on antioxidant enzyme activities and oxidative stability of chicken breast meat. J Agr Food Chem 60: 7111-7120. doi: 10.1021/jf3017207 
3. Arnér ES. 2010. Selenoproteins-what unique properties can arise with selenocysteine in place of cysteine?. Exp Cell Res 316: 1296-1303. doi: 10.1016/ j.yexcr.2010.02.032

4. Bax L. 2016. MIX 2.0 - Professional software for meta-analysis in Excel. Version 2.0.1.5. BiostatXL. [Internet]. Disponible en: https://www.metaanalysis-made-easy.com/

5. Berchieri-Ronchi CB, Kim SW, Zhao Y, Correa CR, Yeum KJ, Ferreira ALA. 2011. Oxidative stress status of highly prolific sows during gestation and lactation. Animal 5: 1774-1779. doi: 10.1017/S1751731111000772

6. Bierla K, Bianga J, Ouerdane L, Szpunar J, Yiannikouris A, Lobinski R. 2013. A comparative study of the $\mathrm{Se} /$ $\mathrm{S}$ substitution in methionine and cysteine in Se-enriched yeast using an inductively coupled plasma mass spectrometry (ICP MS)-assisted proteomics approach. J Proteomics 87: 26-39. doi: 10.1016/ j.jprot.2013.05.010

7. Borenstein M, Hedges LV, Higgins JP, Rothstein HR. 2011. Introduction to metaanalysis. Chichester: John Wiley. 187 p.

8. Bougouin A, Appuhamy JA, Kebreab E, Dijkstra J, Kwakkel RP, France J. 2014. Effects of phytase supplementation on phosphorus retention in broilers and layers: a meta-analysis. Poultry Sci 93: 1981-1992. doi: 10.3382/ps.201303820

9. Catalá-López F, Tobías A. 2014. Metaanálisis de ensayos clínicos aleatorizados, heterogeneidad e intervalos de predicción. Med Clin-Barcelona 142: 270-274. doi: 10.1016/j.medcli.2013.06.013

10. Chen J, Han JH, Guan WT, Chen F, Wang CX, Zhang YZ, Lin, G. 2016a. Selenium and vitamin $E$ in sow diets: I. Effect on antioxidant status and reproductive performance in multiparous sows. Anim Feed Sci Tech 221: 111-123. doi: 10.1016/j.anifeedsci.2016.08.022
11. Chen J, Han JH, Guan WT, Chen F, Wang CX, Zhang YZ, Lin G. 2016b. Selenium and vitamin $\mathrm{E}$ in sow diets: II. Effect on selenium status and antioxidant status of the progeny. Anim Feed Sci Tech 221: 101-110. doi: 10.1016/j.anifeedsci.2016.08.021

12. Chen K, Peng X, Fang J, Cui H, Zuo Z, Deng J, Yang Q. 2014. Effects of dietary selenium on histopathological changes and $T$ cells of spleen in broilers exposed to aflatoxin B1. Int J Env Res Pub He 11: 1904-1913. doi: 10.3390/ ijerph110201904

13. Chew BP. 1996. Importance of antioxidant vitamins in immunity and health in animals. Anim Feed Sci Tech 59: 103-114. doi: 10.1016/03778401(95)00891-8

14. Close WH, Surai PF, Taylor-Pickard $\boldsymbol{J} \boldsymbol{A}$. 2008. Selenium in pig nutrition. Current advances in selenium research and applications. Wageningen: Wageningen Academic. $263 \mathrm{p}$.

15. Collins JF. 2016. Molecular, Genetic and nutritional aspects of major and trace minerals. London: Academic Press. $449 \mathrm{p}$.

16. Dalgaard TS, Briens M, Engberg RM, Lauridsen C. 2018. The influence of selenium and selenoproteins on immune responses of poultry and pigs. Anim Feed Sci Tech 238: 73-83. doi: 10.1016/ j.anifeedsci.2018.01.020

17. Duntas LH, Benvenga S. 2015. Selenium: an element for life. Endocrine 48: 756-775. doi: 10.1007/s12020-0140477-6

18. Egger M, Smith GD, Schneider M, Minder C. 1997. Bias in meta-analysis detected by a simple, graphical test. BMJ 315: 629-634. doi: 10.1136/bmj.315.7109.629

19. Higgins JP, Thompson SG. 2002. Quantifying heterogeneity in a meta analysis. Stat Med 21: 1539-1558. doi: 10.1002/sim.1186 
20. Hu H, Wang M, Zhan X, Li X, Zhao R. 2011. Effect of different selenium sources on productive performance, serum and milk Se concentrations, and antioxidant status of sows. Biol Trace Elem Res 142: 471-480. doi: 10.1007/ s12011-010-8803-1

21. Ighodaro OM, Akinloye OA. 2018. First line defence antioxidants-superoxide dismutase (SOD), catalase (CAT) and glutathione peroxidase (GPX): their fundamental role in the entire antioxidant defence grid. Alexandria J Med 54: 287 293. doi: 10.1016/j.ajme.2017.09.001

22. Kalantari P, Narayan V, Natarajan SK, Muralidhar K, Gandhi UH, Vunta H, Prabhu KS. 2008. Thioredoxin reductase-1 negatively regulates HIV-1 transactivating protein Tat-dependent transcription in human macrophages. J Biol Chem 283: 33183-33190. doi: 10.1074/jbc.M807403200

23. Kim YY, Mahan DC. 2001a. Prolonged feeding of high dietary levels of organic and inorganic selenium to gilts from 25 $\mathrm{kg}$ body weight through one parity. J Anim Sci 79: 956-966. doi: 10.2527/ 2001.794956x

24. Kim YY, Mahan DC. Mahan. $2001 b$. Comparative effects of high dietary levels of organic and inorganic selenium on selenium toxicity of growing-finishing pigs. J Anim Sci 79: 942-948. doi: 10.2527/2001.794942x

25. Krogh U, Bruun TS, Amdi C, Flummer C, Poulsen J, Theil, PK. 2015. Colostrum production in sows fed different sources of fiber and fat during late gestation. Can J Anim Sci 95: 211223. doi: 10.4141/cjas-2014-060

26. Labunskyy VM, Hatfield DL, Gladyshev VN. 2014. Selenoproteins: molecular pathways and physiological roles. Physiol Rev 94: 739-777. doi: 10.1152/ physrev.00039.2013

27. Liu Y, Zhao H, Zhang Q, Tang J, Li K, Xia XJ, Lei XG. 2012. Prolonged dietary selenium deficiency or excess does not globally affect selenoprotein gene expression and/or protein produc- tion in various tissues of pigs. J Nutr 142: 1410-1416. doi: 10.3945/jn.112.159020

28. Luо ZC, Fraser WD, Julien P, Deal CL, Audibert F, Smith GN, Walker M. 2006. Tracing the origins of «fetal origins» of adult diseases: programming by oxidative stress? Med hypotheses 66 : 38-44. doi: 10.1016/j.mehy.2005.08.020

29. Ma YL, Lindemann MD, Pierce JL, Unrine JM, Cromwell GL. 2014. Effect of inorganic or organic selenium supplementation on reproductive performance and tissue trace mineral concentrations in gravid first-parity gilts, fetuses, and nursing piglets. J Anim Sci 92: 5540-5550. doi: 10.2527/jas.20147590

30. Mahan DC. 2000. Effect of organic and inorganic selenium sources and levels on sow colostrum and milk selenium content. J Anim Sci 78: 100-105. doi: 10.2527/2000.781100x

31. Mahan DC, Kim YY. 1996. Effect of inorganic or organic selenium at two dietary levels on reproductive performance and tissue selenium concentrations in first-parity gilts and their progeny. J Anim Sci 74: 2711-2718. doi: $10.2527 / 1996.74112711 \mathrm{x}$

32. Mahan DC, Peters JC. 2004. Longterm effects of dietary organic and inorganic selenium sources and levels on reproducing sows and their progeny. $\mathrm{J}$ Anim Sci 82: 1343-1358. doi: 10.2527/ 2004.8251343x

33. McKenzie RC, Rafferty TS, Beckett GJ. 1998. Selenium: an essential element for immune function. Immunol Today 19: 342-345. doi: 10.1016/S01675699(98)01294-8

34. Mendl M, Zanella AJ, Broom DM. 1992. Physiological and reproductive correlates of behavioural strategies in female domestic pigs. Anim Behav 44: 1107-1121. doi: 10.1016/S0003-3472(05)80323-9

35. Munsterhjelm C, Valros A, Heinonen $M, H a ̈ l l i ~ O$, Siljander-Rasi $H$, Peltoniemi OAT. 2008. Housing during early pregnancy affects fertility and 
behaviour of sows. Reprod Domest Anim 43: 584-591. doi: 10.1111/j.14390531.2007.00956.x

36. National Research Council. 2012. Nutrient requirements of swine. $11^{\text {th }}$ ed. Washington: National Academies Press. $86 \mathrm{p}$.

37. Oropeza-Moe M., Wisloff H, Bernhoft A. 2015. Selenium deficiency associated porcine and human cardiomyopathies. $\mathrm{J}$ Trace Elem Med Bio 31: 148-156. doi: 10.1016/j.jtemb.2014.09.011

38. Pappas AC, Zoidis E, Surai PF, Zervas G. 2008. Selenoproteins and maternal nutrition. Comp Biochem Phys B 151: 361372. doi: 10.1016/j.cbpb.2008.-08.009

39. Phipps RH, Grandison AS, Jones AK, Juniper DT, Ramos-Morales E, Bertin G. 2008. Selenium supplementation of lactating dairy cows: effects on milk production and total selenium content and speciation in blood, milk and cheese. Animal 2: 1610-1618. doi: 10.1017/ S175173110800298X

40. [PIC] Pig Improvement Continuous. 2016. Manual de especificación de nutrientes. Hendersonville. $50 \mathrm{p}$.

41. Quesnel H, Renaudin A, Le Floc'H $N$, Jondreville C, Pere MC, TaylorPickard JA, Le Dividich J. 2008. Effect of organic and inorganic selenium sources in sow diets on colostrum production and piglet response to a poor sanitary environment after weaning. Animal 2: 859-866. doi: 10.1017/ S1751731108001869

42. Rayman MP. 2000. The importance of selenium to human health. lancet 356 : 233-241. doi: 10.1016/S0140-6736(00)02490-9

43. Renaudeau D, Noblet J. 2001. Effects of exposure to high ambient temperature and dietary protein level on sow milk production and performance of piglets. J Anim Sci 79: 1540-1548. doi: 10.2527/ 2001.7961540x

44. Rooke JA, Bland IM. 2002. The acquisition of passive immunity in the new-born piglet. Livest Prod Sci 78: 1323. doi: 10.1016/S0301-6226(02)00182-3
45. Rostagno HS, Teixeira LF, Hannas MI, Lopes J, Sakomura NK, Perazzo FG, Saraiva A, et al. 2017. Tablas brasileñas para aves y cerdos. $4^{\circ}$ ed. Viçosa: Universidad Federal de Viçosa. $444 \mathrm{p}$.

46. Sara A, Odagiu A, Sascã L. 2005. Effects of organic selenium (Sel-plex) administered in reproduction sows on production indices in piglets. Archiva Zootechnica 8: 113-117.

47. Sauvant D, Schmidely P, Daudin JJ, St-Pierre NR. 2008. Meta-analyses of experimental data in animal nutrition. Animal 2: 1203-1214. doi: 10.1017/ S1751731108002280

48. Surai PF. 2006. Selenium in nutrition and health. Nottingham: Nottingham University Press. 120 p.

49. Surai PF, Fisinin VI. 2014. Selenium in poultry breeder nutrition: An update. Anim Feed Sci Tech 191: 1-15. doi: 10.1016/j.anifeedsci.2014.02.005

50. Surai PF, Fisinin VI. 2015. Selenium in pig nutrition and reproduction: boars and semen quality - a review. Asian Austral J Anim 28: 730-746. 10.5713/ ajas. 14.0593

51. Surai PF, Fisinin VI. 2016. Selenium in sow nutrition. Anim Feed Sci Tech 211: 18-30. doi: 10.1016/j.anifeedsci.2015.11.006

52. Svoboda M, Ficek R, Drabek J. 2008. Efficacy of organic selenium from Seenriched yeast on selenium transfer from sows to piglets. Acta Vet Brno 77: 515521. doi: $10.2754 / a v b 200877040515$

53. Thingnes SL, Gaustad AH, Kjos NP, Sandberg E, Framstad T. 2015. The effect of different dietary energy levels during rearing and mid-gestation on sow lifetime performance and longevity. Acta Agr Scand A-An 65: 148-157. doi: 10.1080/09064702.2016.1143962

54. Tsuma VT, Einarsson S, Madej A, Lundeheim N. 1995. Cortisol and âendorphin levels in peripheral circulation around weaning in primiparous sows. Animal Reproduction Science 37(2): 175-182. doi: 10.1016/0378-4320(94)-01330-O 
55. Urso URA, Dahlke F, Maiorka A, Bueno IJM, Schneider AF, Surek D, Rocha, C. 2015. Vitamin E and selenium in broiler breeder diets: effect on live performance, hatching process, and chick quality. Poultry Sci 94: 976983. doi: $10.3382 / \mathrm{ps} / \mathrm{pev} 042$

56. Wang $L, X u X, S u G$ Shi B, Shan A. 2017. High concentration of vitamin $\mathrm{E}$ supplementation in sow diet during the last week of gestation and lactation affects the immunological variables and antioxidative parameters in piglets. $\mathrm{J}$ Dairy Res 84: 8-13. doi: 10.1017/ S0022029916000650

57. Williams AM, Safranski TJ, Spiers $D E$, Eichen PA, Coate EA, Lucy MC. 2013. Effects of a controlled heat stress during late gestation, lactation, and after weaning on thermoregulation, metabolism, and reproduction of primiparous sows. J Anim Sci 91: 2700-2714. doi: 10.2527/jas.2012-6055

58. Wuryastuti H, Stowe HD, Bull RW, Miller ER. 1993. Effects of vitamin E and selenium on immune responses of peripheral blood, colostrum, and milk leukocytes of sows. J Anim Sci 71: 24642472. doi: $10.2527 / 1993.7192464 x$
59. Yoon I, McMillan E. 2006. Comparative effects of organic and inorganic selenium on selenium transfer from sows to nursing pigs. J Anim Sci 84: 1729-1733. doi: $10.2527 /$ jas.2005-311

60. Zhan X, Qie Y, Wang M, Li X, Zhao R. 2011. Selenomethionine: an effective selenium source for sow to improve Se distribution, antioxidant status, and growth performance of pig offspring. Biol Trace Elem Res 142: 481-491. doi: 10.1007/ s12011-010-8817-8

61. Zhao H, Li K, Tang JY, Zhou JC, Wang KN, Xia XJ, Lei XG. 2015. Expression of selenoprotein genes is affected by obesity of pigs fed a highfat diet. J Nutr 145: 1394-1401. doi: 10.3945/jn.115.211318

62. Zhao Y, Flowers WL, Saraiva A, Yeum KJ, Kim, SW. 2013. Effect of social ranks and gestation housing systems on oxidative stress status, reproductive performance, and immune status of sows. J Anim Sci 91: 58485858. doi: $10.2527 /$ jas.2013-6388

63. Zoidis E, Demiris N, Kominakis A, Pappas AC. 2014. Meta-analysis of selenium accumulation and expression of antioxidant enzymes in chicken tissues. Animal 8: 542-554. doi: 10.1017/ S1751731113002395 